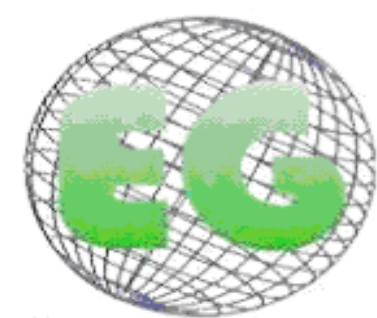

ISSN 1695-6141 $\mathrm{N}^{\circ} 22$
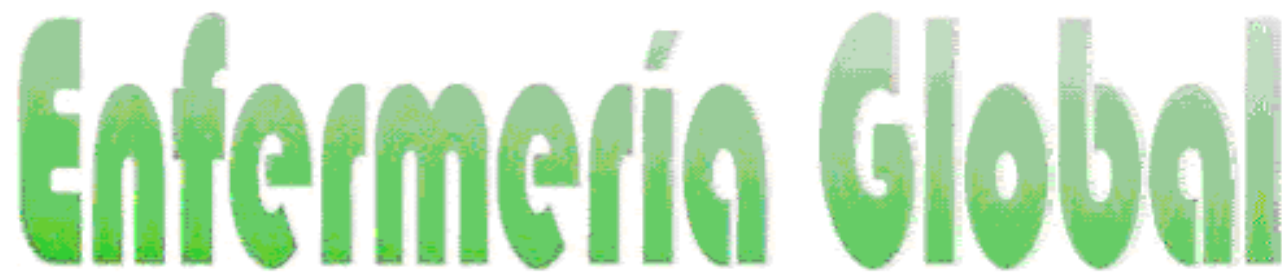

Revista electrónica trimestral de Enfermeria

Abril 2011

www.um.es/egloball

REVISIONES

\title{
IMPORTANCIA DE LA ENFERMERA ESCOLAR EN LA DETECCIÓN DE LA NEOPLASIA TESTICULAR
}

\section{IMPORTANCE OF SCHOOL NURSE IN THE DETECTION OF TESTICULAR NEOPLASIA}

\author{
*Martínez Sabater, A., Sancho Cantus, D., Martínez Puig, C.
}

\author{
`Enfermeros. Hospital Francesc de Borja. Gandia. Valencia. \\ Palabras clave: Enfermera escolar; Neoplasia testicular; Autoexploración testicular \\ Keywords: School nurse; testicular câncer; testicular self-examination.
}

\section{RESUMEN}

En este artículo planteamos una revisión de la literatura en diferentes bases de datos (CINAHL, CUIDEN, MEDLINE) con el fin de valorar la importancia de la neoplasia testicular en la adolescencia y el papel fundamental del profesional de enfermería- concretamente de la enfermera escolar, en la detección precoz de esta patología. Una vez analizado el tema y tras tomar conciencia de la importancia de enseñar prácticas de autoexploración testicular correctas, se ha concluido que la enfermera escolar no debe desarrollar únicamente una actividad asistencial, sino que entre sus funciones se debe contemplar la promoción de la salud y la prevención del cáncer testicular. Dentro del proceso educador, y como fomento de hábitos higiénicos en el adolescente, planteamos dadas las características de presentación de la neoplasia testicular la realización de talleres en los que se instruya a los jóvenes en la autoexploración testicular y la necesidad de realizar una técnica correcta, con el fin de fomentar la adquisición de hábitos de autoexploración testicular al igual que se hace en las mujeres para detectar precozmente la patología mamaria

\section{ABSTRACT}

In this paper we propose a review of the literature in different databases (CINAHL, City, MEDLINE) to assess the importance of testicular cancer in adolescence and the crucial role of the nurse, namely the school nurse, in early detection of this disease. Having analyzed the issue and after becoming aware of the importance of teaching testicular self-examination practices correctly. It was concluded that the school nurse should not only develop a care activity, but that its functions should include the promotion of health and testicular cancer prevention. Within the educational process, and as a promotion for hygiene habits in teenagers, we argue, given the display characteristics of testicular neoplasia conducting workshops to instruct adolescents in testicular self-examination and the need for proper technique, to encourage the acquisition of habits of testicular self-examination like in women for early detection of breast disease 


\section{INTRODUCCIÓN}

La enfermera escolar ha de ser un referente para los alumnos de los centros escolares como educadora en salud e instrucción en hábitos saludables. Dentro de las funciones de la enfermera, la asistencial es solo una de ellas junto con la docente y administradora. A nivel escolar, ha de potenciarse por las características de los discentes, la formación en hábitos saludables de salud: prevención del tabaquismo, dietas saludables, etc., pero también hay que plantear la prevención y la detección precoz de la enfermedad.(1)

Planteamos la necesidad de planificar dentro de las actividades diarias de la enfermera escolar un taller de detección precoz de neoplasias testiculares. Este taller no debe centrarse únicamente en este punto, sino ha de entenderse dentro de una actividad más amplia dentro de un módulo de higiene y conocimiento del propio cuerpo, que permita formar a los alumnos en hábitos de higiene diario, posibles alteraciones (fimosis, frenillo corto...), etc.

\section{MATERIAL Y MÉTODOS}

Se ha realizado una revisión bibliográfica de los aspectos relacionados con la neoplasia testicular en edades preadolescentes y adolescentes, así como la práctica de procedimientos de autoexploración testicular en diferentes bases de datos (CUIDEN; CINAHL...). Como descriptores se han empleado los términos cáncer o neoplasia testicular o autoexploración testicular. Además se han empleado los conectores booleanos AND, OR. Se han eliminado aquellos términos que pudieran haber producido confusión o ruido en la búsqueda (salud adolescente, por ejemplo).

\section{RESULTADOS}

Los tumores testiculares representan aproximadamente el $1 \%$ de las neoplasias masculinas, siendo más frecuente entre los 15 y 35 años ${ }^{(2)}$. En el estudio de López-Abente y colaboradores $^{(3)}$ estimaban una incidencia bruta anual de 820 casos de neoplasia testicular con una tasa ajustada a la población europea de 3.85. Otros estudios revisados como el de Cáceres C.A. et $\mathrm{al}^{(4)}$, establecen una incidencia poco frecuente (1-2 casos por 100.000 habitantes). La base de datos Globocan $2002^{(5)}$ indica en España en el años 2005 la aparición de 418 casos nuevos de cáncer testicular que representan el $0.43 \%$ de los tumores masculinos, con una mortalidad total de 37 casos $(0.06 \%$ de las muertes). Esta tendencia, tal como aparece en el estudio de LLanes et al ${ }^{(6)}$ en España, al igual que en otros países desarrollados, se está produciendo un aumento de la incidencia del cáncer testicular. Pese a encontrarnos con un tipo de patología con un alto grado de curación en las fases tempranas, sigue siendo frecuente encontrar casos en la bibliografía en los que el diagnóstico es tardío y la consulta es realizada por la presencia de sintomatología metastásica ${ }^{(, 7,8,9,10,11)}$.

En el estudio publicado por la profesora Rojo(12), vemos la importancia y el papel del profesional de enfermería en la prevención del cáncer testicular mediante la autoexploración testicular regular, fomentando la educación sanitaria y protocolizando la autoexploración testicular. Incluso estudios como el de Maloret de $2007^{(13)}$ van más allá y fomentan la autoexploración en pacientes con dificultades de aprendizaje con el fin de mejorar el diagnóstico y la supervivencia. Diferentes estudios valoran la idoneidad de la autoexploración testicular como promotor de hábitos saludables ${ }^{(14)}$, no obstante hay que tener en cuenta que hemos encontrado una revisión sistemática ${ }^{(15)}$ donde no se encuentra evidencia respecto a la educación en cuanto a que el autoexamen mejore el riesgo, no 
obstante, se indica en el artículo que dadas la baja incidencia de casos y la buena respuesta de la patología pese a la ausencia de screening, hace que la autoexploración pueda proporcionar mayores beneficios. Otra revisión sistemática posterior ${ }^{(16)}$ sugiere que el autoexamen testicular y el screening mediante ecografia testicular podrían ser métodos efectivos para la detección precoz de este cáncer.

Por otro lado, estudios como el de McCullagh ${ }^{(17)}$ evidencian la mejora tras un estudio de casos-control postintervención en el puesto de trabajo del porcentaje de autoexploración. Respecto a la información, el trabajo de Rudberg ${ }^{(18)}$ en Suecia, tras el análisis del cuestionario suministrado a los alumnos y en el que entre otras cosas indican que su principal fuente de información respecto a la patología sería los medios de comunicación, concluye que es necesario educar en salud respecto al tema, y considera que sería idóneo realizarlo en la escuela y durante el servicio militar. Otros estudios plantean que esta formación se realice en colaboración con organizaciones juveniles ${ }^{(19)} \mathrm{o}$ en la escuela, con la colaboración del profesor de gimnasia ${ }^{(20)}$

Respecto a estudios de mantenimiento de hábitos de autoexploración, el estudio de Lechner de $2002{ }^{(21)}$ indica que solo un $2 \%$ de los encuestados realizan regularmente autoexploración testicular, e indica que realizar intervenciones educativas en la escuela favorecerá tanto la implantación de hábitos como el mantenimiento posterior. Es importante, en nuestra opinión, tanto la enseñanza como el mantenimiento, así en el estudio de Brenner J. et al, ${ }^{(22)}$ se realiza una valoración respecto a la capacidad educativa de los Residentes de Pediatría en Estados Unidos respecto a la autoexploración; solo el $29 \%$ de los residentes realizan autoexploración (contestan mayoritariamente que se les olvida realizársela)

\section{DISCUSIÓN}

Consideramos que la escuela va a ser el lugar idóneo para la realización de las intervenciones que se han ido explicando. En la Agencia Valenciana de Salut desde el año 1986 (con el decreto 147/86) ${ }^{(23)}$ se iniciaba el Programa de Salud del Niño, y en el artículo 19 se creaba la Comisión para la promoción de la salud del niño en la escuela. No obstante, en España, como dice el artículo de Davó y colaboradores ${ }^{(24)}$, los programas de educación tienen una vertiente más preventiva que de promoción.

Dada la importancia vista en la revisión bibliográfica de la Autoexploración testicular, nos planteamos desarrollar una intervención educativa en la Escuela con el fin de facilitar en el alumno la asunción de la importancia de realizar una correcta técnica autoexploratoria.

La intervención educativa irá encaminada a alumnos de Cuarto curso de E.S.O. (16 años). Dadas las características del tema, consideramos necesaria la colaboración del profesor tutor o el profesor de Educación Física, colaboración que en la bibliografía consultada indica un aumento del porcentaje de éxito

En primer lugar, planteamos una encuesta previa para conocer hábitos exploratorios (frecuencia, etc.) e información previa recibida. Al mismo tiempo, nos planteamos la necesidad, con el fin de evitar cuadros de ansiedad/pánico por "miedo cáncer", introducir la técnica dentro de una charla más amplia de Higiene Genital (hábitos, patologías (frenillo corto, fimosis, etc.)

Iniciaremos con una charla con la anatomía normal del Aparato genital masculino. Se utilizará Maniquí modelo para la exploración testicular, con el fin de que puedan identificar las diferentes estructuras que componen el aparato reproductor (saco escrotal, epídimo, 
conducto deferente, testículo), nos ayudaremos con vídeos y otros medios audiovisuales con el fin de visualizar la correcta técnica, teniendo en cuenta incidir en:

1. Realizar exploración después de la ducha con agua caliente para favorecer la dilatación escrotal.

2. Realizar exploración mensual, buscando el momento adecuado (tranquilidad, intimidad, etc.)

3. Explorar cada testículo por separado y valorar textura, tamaño y color.

4. Conocer los signos de alarma: cambio de tamaño o consistencia, bulto o nódulos, etc.

5. Incidir en la importancia de asistir al personal del Centro de Atención Primaria.

\section{CONCLUSIÓN}

Aunque la incidencia de las neoplasias testiculares es mucho más pequeña que la de otros tipos (mama, ovario, próstata, etc.) en el que desde hace años se han planteado campañas de prevención, screening y detección precoz, el impacto que suele tener en los individuos afectados y en su círculo cercano, por la edad de aparición, etc., hace necesario el formar a los jóvenes en las características de su cuerpo y las posibles alteraciones. En el caso de este tipo de patología, la detección precoz y el conocimiento de la importancia de esta, puede ser un factor importante para evitar complicaciones tardías y para la adopción de hábitos exploratorios.

Como se ha comprobado en la bibliografía existe en diferentes situaciones una falta de información por parte de los jóvenes, al igual que una falta de hábito exploratorio que puede suponer un retraso en el diagnóstico precoz. La existencia de una enfermera escolar debe ser un referente para el alumno que le ayude a solucionar dudas en temas relacionados con la salud y la enfermedad en general, y en relación con la patología neoplásica, en particular.

\section{BIBLIOGRAFÍA}

1. Ortiz Vela M. et al. Perfil profesional de la Enfemera escolar.Disponible en http://www.sce3.com/docs/perfil enf escolar gandia 2009.pdf (consultado el 16/05/10)

2. Mantilla T., Alda de la Fuente V. Tumores testiculares en Atención Primaria. A proposito de dos casos. Semergen 24 (1): 28-30.

3. Lopez-Abente G. Et al Situación del cáncer en España. Incidencia. An. Sist. Sanit. Navar. 2004; 27 (2): 165-173

4. Cáceres Saglio C.A, Hortensia M, Bresanovich V.A. Mandarino A. Tumor testicular: tumor de Yolk Sac. Presentación de un caso clínico. Revista de posgrado de la Via Cátedra de Medicina. №149 Septiembre 2005

5. GLOBOCAN 2002 database: Url: http://www-dep.iarc.fr Consultado el 18 de Mayo de 2009

6. Llanes Gonzalez, $L$ et al. Tendencia en la incidencia del cáncer testicular de células germinales en un área sanitaria española de 300.000 habitantes (1991-2005). Actas Urol. Esp 2008;32(7):691-695

7. Pastor Navarro, H. Et al. Hemoperitoneo y muerte por rotura de metástasis hepatoesplénicas de tumor testicular. Actas Urol. Esp. 2007; 31 (10) 1175-1178

8. Avasthi R., et açl Testicular mixed germ cell tumor metastasizing to Herat. J Assoc Physicians Incia 2008 Oct; 56:812-5 
9. Shahrokh F Gastrointestinal hemorrhage as first manifestation of metastasic testicular tumor. Urology Volume 66 Issue 6, December 2005, 1319 21-24

10. Chhieng D, Jennings T., Slominski A., Martin C., Mihm Jr. Choriocarcinoma presenting as a cutaneous metastasis Journal of Cutaneous Pathology Published Online: 27 Apr 2006

11. Molina Infante J. et al Hemorragia digestive secundaria a coriocarcinoma con metastasis gástricas y colónicas. Rev.Esp.Enf.Digestivas 2004; 96(1):77-80

12. Rojo Pascual M.C, et al Actuación enfermera en la prevención del cáncer testicular. Metas de Enfermería. Número 43, Marzo 2002

13. Maloret $\mathrm{p}$. Testicular self-examination: the person with learning dificultéis $\mathrm{Br} \mathrm{J}$ Nurs 2007 Aug 9-Sep 12; 16(15): 931-5

14. Roemer HC, et al. Palpation of the testes in draftees: acceptance on the occasion of muster and implication for health promotion. Int J Occup Med Envron Health 2006; 19 (4):254-9

15. Calonge N. Screening for testicular cancer: recommendation statement Am Farm Physician 2005 Nov 15; 72(10): 2069-70

16. Cambil Martín J. Efectividad del autoexamen y del screening en la detección del cáncer de testículo. Index Enferm. [periódico en Internet]. 2005 Jun [citado 2009 Mayo 19] ; 14(48-49): 33-36. Disponible en: http://scielo.isciii.es/scielo.php?script=sci_arttext\&pid=S1132$12962005000100007 \&$ Ing=es\&nrm=iso.

17. McCullagh J. Promoting awareness and practice of testicular self-examination Nurs Stand 2005 Aug 31 - Sep 6; 19(51):41-9

18. Rudberg L. et al Testicular cancer and testicular self-examination: knowledge an attitudes of adolescent Swedish men. Cancer Nurs, 2005 Jul-Aug; 28(4):256-62

19. Ward Kd. Et al Testicular cancer awareness and self-examination among adolescents males in a community-based youth organization. Prev. Med 2005 Aug; 41(2); 386-98

20. Zientek CE, Dewald LL. Cancer detection: The educational role of the Athletic trainer. J Ath Train 1998 Jan; 33(1) 45-49

21. Lechner L. Oenema A, de Nj. Testicular-self examination (TSE) among Dutch young men aged 15-19: determinants of the intention to practice TSE. Health Educ Res 2002 Feb; 17(1): 73-84

22. Brenner J.S., Hergenroeder A., Kozinetz C.A., . Kelder SH Teaching Testicular SelfExamination: Education and Practices in Pediatric located on the World Wide Web at: http://www.pediatrics.org/cgi/content/full/111/3/e239 DOI: $10.1542 /$ peds.111.3.e239 Pediatrics 2003;111;e239-e244

23. Los equipos de Atención Primaria y su intervención en la Escuela. Edita Conselleria de Sanitat. Dirección General de Salud Pública. Disponible en: http://biblioteca.sp.san.gva.es/biblioteca/publicaciones/MATERIAL/PUBLICACIONES/ PROMO SALUD/EDUCACION/ESCUELA/LOS EQUIPOS DE AP.PDF Consultado el $28 / 05 / 2009$

24. Davó Blamnes, MC. Gil D., Vives C. Alvarez-Dardet C., La Parra, D. Las investigaciones sobre promoción y educación para la salud en las etapas de infantil y primaria de la escuela española: una revisión de los estudios publicados entre 1995 y 2005. Gaceta Sanitaria. Vol 22 nำ1 2008 58-64 